\title{
Determinantes biopsicossociais que predizem qualidade de vida em pessoas que vivem com HIV/AIDS
}

\author{
Bruno Medeiros \\ Universidade de Cambridge \\ Josevânia da Silva \\ Centro Universitário de João Pessoa \\ Ana Alayde Werba Saldanha \\ Universidade Federal da Paraíba
}

\begin{abstract}
Resumo
$\mathrm{O}$ advento da terapia antirretroviral trouxe a necessidade de se compreender os determinantes psicossociais envolvidos na avaliação de qualidade de vida em pessoas que vivem com HIV/AIDS. O objetivo desse estudo é investigar os determinantes psicossociais e clínicos envolvidos na avaliação de qualidade de vida nesse grupo social. Esta pesquisa envolveu 90 pessoas vivendo com HIV/AIDS (média de idade de 33,7 anos, $D P=6,6)$. Um questionário sócio-demográfico e clínico e o WHOQOL-BREF constituíram os principais métodos. Análises descritivas, comparações entre médias de grupos-critério e análise de regressão foram utilizadas. Os resultados demonstram melhor qualidade de vida entre os que estavam satisfeitos com os serviços de saúde do hospital, bem como os principais determinantes para a avaliação de qualidade de vida são a dimensão psicológica, contagens de células CD4 e a dimensão ambiental. Essa pesquisa sugere a elaboração de políticas públicas de saúde em HIV/AIDS que englobem os fatores psicossociais.
\end{abstract}

Palavras-chaves: qualidade de vida; HIV/AIDS; determinantes psicossociais; CD4.

\begin{abstract}
Biopsychosocial determinants that predict quality of life in people living with hiv/AIDS. The advent of the antiretroviral therapy highlighted the need to understand psychosocial determinants of quality of life assessment in people living with HIV/AIDS. The purpose of this study was to investigate the psychosocial and clinical determinants related to the quality of life of this group. This investigation involved 90 patients (age mean 33.7 years old, $S D=6.6$ ). Socio-demographic questions, a clinic questionnaire, and the instrument for assessment of quality of life WHOQOL-BREF were the main methods. Descriptive statistics, comparison between means of the criterion groups, and a regression were the main analyses. The results demonstrate better assessment of quality of life in patients who were satisfied with health care provided by the hospital. Psychological dimension, CD4 cells count, and environmental factors are the main determinants to the quality of life assessment. This research recommends the elaboration of health public politics toward HIV/ AIDS that include psychosocial aspects.
\end{abstract}

Keywords: quality of life; HIV/AIDS; psychosocial determinants; CD4.

\section{Resumen}

Determinantes biopsicosociales que predicen la calidad de vida en personas con vih/sida. La aparición de la terapia antiviral destacó la necesidad de entender los determinantes psicosociales en la evaluación de la calidad de vida de las personas viviendo con VIH/SIDA. El objetivo de este estudio es investigar los determinantes clínicos y psicosociales relacionados con la calidad de vida de este grupo. Esta investigación involucra a 90 pacientes (edad media 33,7 años, desviación típica 6,6). Los principales métodos utilizados fueron preguntas socio-demográficas, un cuestionario clínico, y el instrumento de evaluación de calidad de vida WHOQOL-BREF. Los principales análisis consistieron en estadísticas descriptivas, comparaciones entre las medias de los grupos de criterio, y una regresión. Los resultados muestran una mejor evaluación de la calidad de vida en los pacientes que estaban satisfechos con la atención médica provista por sus hospitales. La dimensión psicológica, el recuento de células CD4, y los factores medioambientales son los determinantes 
más importantes para la evaluación de la calidad de vida. Esta investigación recomienda la elaboración de políticas públicas sanitarias en torno al VIH/SIDA que tengan en cuenta los aspectos psicosociales.

Palabras clave: calidad de vida; VIH/SIDA; determinantes psicosociales; CD4.

$\mathrm{A}$ avaliação do impacto do vírus da imunodeficiência humana (HIV) na saúde e na qualidade de vida das pessoas tem sido objeto de estudos desde o início da epidemia. Nas décadas de 1980 e 1990 o foco dos estudos se deu nos aspectos clínicos e epidemiológicos da infecção, bem como nas implicações negativas da AIDS na saúde física e mental (Almeida \& Labrinici, 2007; Fleck, 2008; Guimarães, 2001; Zimpel \& Fleck, 2008). Na contemporaneidade, com o aumento da expectativa de vida entre pessoas que vivem com a AIDS, a investigação sobre qualidade de vida se tornou ainda mais relevante devido à busca de instrumentos de avaliação de saúde mais abrangentes, ou seja, que envolvam fatores psicológicos, sociais e estruturais (Chatterji \& Bickenbach, 2008; Panzini, Rocha, Bandeira, \& Fleck, 2008). Assim, busca-se compreender como essas pessoas vivenciam o maior prolongamento de vida decorrente, em parte, dos efeitos da Terapia Antirretroviral (TARV). Nesse sentido, procura-se analisar os aspectos biopsicossociais envolvidos na avaliação e satisfação com a vida de indivíduos que vivem com HIV/AIDS na atualidade.

A mudança do foco de estudos, bem como a inclusão da AIDS na categoria de doença crônica, trouxe a procura pela promoção de qualidade de vida nas políticas públicas e órgãos de saúde. Nessa direção, a Organização Mundial de Saúde, através do grupo WHOQOL, define qualidade de vida como "a percepção do indivíduo de sua posição na vida, no contexto da cultura e sistema de valores nos quais ele vive e em relação aos seus objetivos, expectativas, padrões e preocupações" (The WHOQOL Group, 1995, p.1405). Esse é um conceito amplo e abarca uma variedade de condições que podem influenciar a forma como o indivíduo percebe o seu funcionamento diário, podendo afetar seus sentimentos e comportamentos, mas não se limitando à sua condição de saúde física. Assim, muda-se o foco dos estudos, antes centrado na cura e na medicalização da vida, para o viver com qualidade mesmo diante de doenças crônicas (Ravagnani, Domingos, \& Miyazaki, 2007).

No contexto de pessoas que vivem com a AIDS, a percepção de qualidade de vida satisfatória tem sido relacionada com funcionamento psicológico, suporte social, capacidade física e religiosidade (Fleck et al., 1999; Santos, França Junior, \& Lopes, 2007). Estudos sobre a relação entre determinantes psicossociais e qualidade de vida em pessoas com AIDS têm levantado importantes dimensões no enfrentamento dessa doença (Seidl, 2005). Por exemplo, Remor (2002) revelou que o apoio social contribuiu para o enfrentamento à mudanças, diminuição de sintomas de ansiedade e melhores avaliações de qualidade de vida entre pacientes de um hospital universitário em La Paz, Madri. A dimensão de suporte social foi evidenciada em outro estudo na China (Meng et al., 2008). Nessa pesquisa, pessoas vivendo com HIV/AIDS apresentaram menor apoio social e obtenção de trabalho estável em comparação com a população geral investigada, sugerindo que experiências de estigma e discriminação contribuíram para uma avaliação de vida desfavorável. No Brasil, em Porto Alegre, uma melhor avaliação geral de qualidade de vida esteve associada à diminuição de sintomas depressivos e de ansiedade, contudo, pacientes com AIDS apresentaram maiores índices de depressão (Zimpel \& Fleck, 2008). Esses autores não apenas ressaltam a inter-relação entre determinantes clínicos e psicossociais na vivência do HIV/ AIDS, mas a necessidade de se incluir a avaliação do sistema imunológico em estudos de qualidade de vida no contexto do HIV.

Estudos têm apontado a relação entre os níveis de linfócitos CD4 (células que regulam o sistema imunológico), aspectos psicológicos e dimensões da qualidade de vida (Ironson et al., 2005; Torres \& Oliveira, 2004). Baixos níveis de CD4 têm sido apontados como fatores de risco para o surgimento de, por exemplo, quadros de demência - característico do estágio mais grave dos transtornos cognitivos -, acometendo cerca de $5 \%$ dos indivíduos já na fase sintomática da infecção, ou seja, quando há um comprometimento da resposta imune (Torres \& Oliveira, 2004).

No que se refere à dimensão psicológica, a literatura demonstra que determinados estados psíquicos como depressão e ansiedade podem influenciar o sistema imunológico (Remor, 2002; Zeña et al., 2009). Não se quer afirmar, no entanto, que características emocionais e psicológicas sejam a causa de patologias, mas que condições de estresse percebido, ansiedade, depressão, entre outras, podem contribuir para a diminuição da resposta imune do organismo, o que pode ocasionar o surgimento de doenças oportunistas, trazendo implicações para a saúde de pessoas que vivem com HIV/AIDS. (Remor, Penedo, Shen, \& Schneiderman, 2007).

Já em relação aos aspectos clínicos decorrentes da infecção pelo HIV, segundo o Ministério da Saúde do Brasil (2005), com o advento da TARV as manifestações clínicas (como o aparecimento de doenças oportunistas) tornaram-se menos frequentes e houve melhora substancial do prognóstico e da percepção de qualidade de vida dos indivíduos. O principal objetivo da TARV seria, através da inibição da replicação viral, retardar o progresso da imunodeficiência e restaurar, tanto quanto possível, a imunidade, contribuindo para o aumento da sobrevida das pessoas. Assim, no que se refere aos aspectos clínicos, verifica-se que o uso combinado de três ou mais drogas antirretrovirais prolonga a sobrevida das pessoas e pode também reduzir a incidência de transtornos psicológicos associados ao HIV (McCutchan et al., 2007), estando tal redução estritamente relacionada com o tempo decorrido para o início do tratamento e a adesão ao mesmo no decorrer do processo.

Entretanto, não apenas fatores subjetivos e clínicos estão associados ao enfrentamento e progressão do HIV/AIDS. Aspectos estruturais como pobreza econômica e desigualdades sociais também têm se constituído fatores importantes não só 
para as pessoas que vivem com o HIV/AIDS (Seidl, Melchíades, Farias, \& Brito, 2007), mas também estão relacionados com os índices epidemiológicos que caracterizam os quadros de notificação do vírus (Parker \& Camargo Jr, 2000). No quadro de estudos em qualidade de vida e saúde, o domínio ambiental satisfação com acesso a serviços de saúde, recursos financeiros, acesso à informação, qualidade de moradia, entre outros. constitui importante fator para uma avaliação satisfatória de saúde (Fleck, 2008, Fleck et al., 1999). Nesse sentido, acesso público à terapia antirretroviral e melhor eficácia do tratamento tem se relacionado a melhores avaliações de qualidade de vida em pessoas que vivem com AIDS (Santos Souza \& Lopes, 2007). Por outro lado, em outro estudo conduzido por Seidl, Zannon e Trócolli (2005), escassez de recursos financeiros e segurança física foram relacionados a avaliações negativas no domínio ambiental de qualidade de vida. Segundo estes autores, esses resultados sugerem a necessidade de se investigar não apenas as questões individuais e biológicas relacionadas à epidemia, mas também a interação entre os aspectos psicológicos, sociais e estruturais de risco.

Embora se considere a relação entre qualidade de vida e os aspectos supracitados, há que se verificar, ainda, de que forma se dá essa relação, ou seja, quais variáveis estão mais presentes na avaliação de qualidade de vida por parte das pessoas que vivem com o HIV/AIDS. Devido ao aspecto multidimensional da qualidade de vida, esse estudo propõe que as dimensões ambiental e psicológica assumem importância na avaliação da qualidade de vida, principalmente em pessoas que vivem com HIV/AIDS. De igual modo, sugere-se que essa avaliação seja feita se levando em conta o estado de saúde do indivíduo, bem como a carga viral e a contagem de células CD4. Portanto, a identificação das variáveis explicativas da qualidade de vida dessa categoria social poderá informar modelos de intervenção psicossocial. Nesse sentido, o objetivo desse estudo foi investigar os determinantes psicossociais e clínicos envolvidos na qualidade de vida em pessoas que vivem com HIV/AIDS.

\section{Método}

\section{Característica do estudo}

Trata-se de estudo transversal, podendo ser considerado como um estudo epidemiológico descritivo, cujo design possibilita a determinação da prevalência das variáveis estudadas.

\section{População/Participantes}

A população deste estudo consistiu de pessoas que vivem com HIV/AIDS, na faixa etária entre 18 anos e 50 anos atendidas em um serviço ambulatorial de referência à AIDS na cidade de João Pessoa, Paraíba, Brasil. A escolha por esta faixa etária se deu em função desse segmento populacional concentrar um número de pessoas aptas para desenvolver atividades produtivas. O planejamento amostral foi efetuado considerando um intervalo de confiança de $95 \%$; erro de $2 \%$, de forma não probabilística e acidental, ficando a amostra constituída por 90 pessoas que vivem com HIV/AIDS na faixa etária de 19 a 48 anos, as quais recebem atendimento de referência em relação à soropositividade ao HIV/AIDS.

Foram elegíveis para a pesquisa pessoas com exame confirmatório de infecção pelo HIV no prontuário médico; idade igual ou superior a 18 anos e até 50 anos; que não tinham dificuldade de comunicação que comprometesse o entendimento do questionário; e participação voluntária no estudo assinando o Termo de Consentimento Livre e Esclarecido. Foram adotados os seguintes critérios de exclusão: recusa a participar do estudo; ausência de informações importantes no questionário; e pessoas acometidas de transtornos mentais graves ou com agravamento do quadro de saúde.

\section{Instrumentos}

Para a coleta de dados foram utilizados os seguintes instrumentos:

1. Questionário sócio-demográfico e clínico, com o objetivo de caracterizar os participantes com questões versando sobre: sexo, idade, grau de escolaridade, renda familiar, situação conjugal, situação empregatícia, religiosidade e dados clínicos (tempo de diagnóstico do vírus, quantidade de células CD4, tratamento medicamentoso, ocorrência de doenças oportunistas, etc.).

2. Escala de Avaliação de Qualidade de Vida (WHOQOLBREF ), instrumento construído pela Organização Mundial de Saúde e validado para a população brasileira por Fleck et al.(1999), que considera a qualidade de vida um construto subjetivo (percepção do indivíduo em questão), multidimensional (dimensões psicológicas, estruturais, ecológicas etc.) e composto por elementos positivos (por exemplo, mobilidade) e negativos (dor). O WHOQOL-BREF deriva do WHOQOL-100 - a forma mais ampliada do instrumento - e possui 26 questões englobando quatro domínios: a) físico; b) psicológico; c) relações sociais e d) meio ambiente. O instrumento, utilizando uma amostra heterogênea de pacientes com diferentes doenças e tratados tanto em regime ambulatorial como hospitalar, mostrou características satisfatórias de consistência interna (Coeficientes de Cronbach de 0,69$)$, validade discriminante $(p<0,01)$ e fidedignidade testereteste (Coeficientes de correlação entre 0,69 a 0,81).

\section{Procedimentos}

Após prévia autorização do Comitê de Ética em Pesquisa da Secretaria de Saúde do Estado da Paraíba, bem como da instituição pesquisada, foi realizada a etapa de coleta de dados no período de abril, 2009. A aplicação dos questionários foi individual, realizado pelo pesquisador em uma sala reservada do ambulatório hospitalar, sendo solicitado aos participantes que assinassem o Termo de Consentimento Livre e Esclarecido, cujo modelo foi elaborado de acordo com a Resolução nº 196/96 Sobre Pesquisa Envolvendo Seres Humanos (Brasil, 1996). Se os participantes não soubessem ler, o(a) pesquisador(a) teria a responsabilidade de ler e explicar o Termo de Consentimento Informado, deixando claras as suas intenções ao realizar a pesquisa, assim como ler e explicar as questões dos instrumentos para que os mesmos pudessem ser respondidos pelos participantes.

\section{Análise dos dados}


Os dados sócio-demográficos foram submetidos à análises descritivas. Os escores do instrumento WHOQOL-BREF foram transformados em índices com ponderação de 0 a 100 , em que 0 corresponde ao menor e 100 ao maior valor encontrado para cada domínio de qualidade de vida, atendendo assim, aos critérios psicométricos de mensuração da escala que propõe um ponto de corte acima de 50 para avaliações positivas e abaixo de 50 para avaliações negativas acerca da qualidade de vida. A consistência interna dos fatores para a amostra em estudo, medido pelo alpha de Cronbach, mostra-se satisfatória (físico $=0,72 ;$ psicológico $=$ 0,68 ; relações sociais $=0,60$; meio-ambiente $=0,65$ ).

Com a utilização do software SPSS, versão 15 , os dados foram analisados utilizando-se o teste $t$ de Student para comparações de médias. O coeficiente de correlação de Pearson foi calculado entre os domínios (físico, ambiental, social e psicológico) e a qualidade de vida global. Modelos de análises de regressão linear múltipla (stepwise) foram construídos para verificar a contribuição das variáveis na qualidade de vida global. O nível de significância utilizado foi de $5 \%$.

\section{Resultados}

Dos participantes entrevistados, $56 \%$ eram do sexo masculino, com média de idade de 33,7 anos $(D P=6,6-$ variando de 19 a 48 anos), com baixa renda (81,1\% recebiam até dois salários mínimos; $10 \%$ recebiam de dois até cinco salários; e $8,9 \%$ dos entrevistados recebiam mais que cinco salários) e baixa escolaridade ( $64 \%$ estudo até o Ensino Fundamental). Quanto à atividade laboral, 52\% dos participantes estavam empregados, enquanto que $33 \%$ não possuíam emprego e $14 \%$ recebiam aposentadoria ou auxílio doença. A maioria residia na área metropolitana (63\%), 30\% moravam em cidades do interior e $7 \%$ em outros Estados.

Em relação à religião, $60 \%$ dos pesquisados se autodenominaram católicos, $18 \%$ evangélicos, $11 \%$ mencionaram pertencerem a outra religião/forma de espiritualidade e $11 \%$ afirmaram não ter religião. Com relação à avaliação do quanto os participantes se consideravam religiosos a maioria se denominou "Religiosa" (33\%) e "Muito Religiosa" (30\%).

Quanto à caracterização clínica dos participantes, a média do tempo de diagnóstico foi de 6,2 anos, $(D P=4,91$ - variando de 1 até 28 anos), $88 \%$ dos entrevistados estavam em uso da terapia antirretroviral e $60 \%$ relataram não terem contraído doenças oportunistas. A contagem dos linfócitos CD4 (indicador das condições do sistema imunológico) por milímetro cúbico de sangue, verificada nos prontuários, variou desde 32 até 1250 CD4/ $\mathrm{mm}^{3}$, apresentando uma média de $557,42 \mathrm{CD} 4 / \mathrm{mm}^{3}$ (DP $=248,187)$.

A percepção dos pesquisados acerca da sua qualidade de vida geral foi positiva $(M=65 ; D P=19,9)$, bem como em relação aos seus domínios, a saber: físico $(M=59,56 ; D P=$ $17,81)$, psicológico $(M=70,64 ; D P=15,61)$, social $(M=65,83$; $D P=22,72)$ e meio ambiente $(M=56,66 ; D P=15,75) . \mathrm{Na}$ análise por domínios associados aos dados sócio-demográficos, as mulheres avaliaram melhor o domínio físico de qualidade de vida que os homens $(t=2,29 ; g l=82,9 ; p=0,02)$. Em relação aos dados clínicos, ao se verificar a relação entre contagem de células CD4, presença de sintomas e percepção de qualidade de vida nas pessoas que vivem com HIV/AIDS, constatou-se que os pacientes com uma contagem de linfócitos T, CD4 acima de 500 por $\mathrm{mm}^{3}$ avaliaram melhor a sua vida que os pacientes com um contagem abaixo de $500 \mathrm{~mm}^{3}(t=2,18 ; g l=31 ; p=0,03)$.

A avaliação do acesso aos serviços de saúde utilizados aponta para 67\% dos participantes "Satisfeitos" ou "Muito Satisfeitos" com o acesso aos serviços de saúde que utilizavam. Foi verificado que os usuários que mais se consideraram satisfeitos com o acesso aos serviços de saúde apresentaram maiores médias na avaliação de qualidade de vida geral que os insatisfeitos $(t=2,76$; $g l=88 ; p=0,007)$, bem como apresentaram melhores avaliações nos domínios: físico $(t=2,45 ; g l=88 ; p=0,01)$, psicológico $(t$ $=2,89 ; g l=88 ; p=0,005)$ e ambiental $(t=5,59 ; g l=59,28 ; p=$ 0,001). Esses resultados são explicitados na Tabela 1:

Tabela 1

Domínios da Qualidade de Vida e Qualidade de Vida Geral em Relação à Satisfação com o Acesso aos Serviços do Hospital (Teste t de Student).

\begin{tabular}{|c|c|c|c|c|}
\hline Domínios da Qualidade de Vida & Grupos critérios & Média & Desvio padrão & $p$ \\
\hline Físico & $\begin{array}{l}\text { Menos Satisfeito } \\
\text { Mais Satisfeito }\end{array}$ & $\begin{array}{l}53,21 \\
62,73\end{array}$ & $\begin{array}{l}18,24 \\
16,85\end{array}$ & 0,01 \\
\hline Psicológico & $\begin{array}{l}\text { Menos Satisfeito } \\
\text { Mais Satisfeito }\end{array}$ & $\begin{array}{l}64,16 \\
73,88\end{array}$ & $\begin{array}{l}16,93 \\
13,95\end{array}$ & 0,005 \\
\hline Ambiental & $\begin{array}{l}\text { Menos Satisfeito } \\
\text { Mais Satisfeito }\end{array}$ & $\begin{array}{l}45,31 \\
62,34\end{array}$ & $\begin{array}{l}13,40 \\
13,70\end{array}$ & 0,001 \\
\hline Qualidade de Vida Geral & $\begin{array}{c}\text { Menos Satisfeito } \\
\text { Mais Satisfeito }\end{array}$ & $\begin{array}{l}57,08 \\
68,95\end{array}$ & $\begin{array}{l}20,41 \\
18,62\end{array}$ & 0,007 \\
\hline
\end{tabular}

* significativo a $p \leq 0,05$

A correlação de Pearson entre os domínios (físico, psicológico, social e ambiental) e o domínio global da qualidade de vida indicou que todos os domínios se correlacionaram positiva e significativamente com o domínio global, assim como os domínios entre si, exceto o domínio relações social com o domínio físico (considerando correlações acima de 0,35). Esses 
resultados podem ser verificados na Tabela 2:

Procedeu-se à análise de regressão linear múltipla (stepwise) para se verificar a contribuição de cada domínio e variáveis na qualidade de vida global (variável dependente). Dentre os quatro domínios apenas o psicológico e o ambiental entram no modelo explicativo, acrescido da variável CD4 (Tabela 3). Juntos

Tabela 2

Estimativa dos Coeficientes de Correlação de Pearson entre a Qualidade de Vida Global (QV) e seus domínios (N=90).

\begin{tabular}{lllll}
\hline Variáveis & QV & DF & DP & DRL \\
\hline D. Físico & 0,483 & & & \\
D. Psicológico & 0,000 & & & \\
& 0,629 & 0,545 & 0,513 & \\
D. Relações Sociais & 0,000 & 0,000 & 0,000 & 0,458 \\
D. Ambiental & 0,480 & 0,001 & 0,473 & 0,000 \\
\hline
\end{tabular}

* significativo a $p \leq 0,05$

explicam $51 \%$ da qualidade de vida global. O domínio que mais contribui na qualidade de vida global é o psicológico $(26 \%)$, seguido da variável CD4 (15\%) e do Domínio Ambiental (10\%).

Tabela 3

Análise de Regressão Múltipla com as Variáveis que Mais Explicaram a Qualidade de Vida Geral.

\begin{tabular}{lccccccc}
\hline VARIÁVEIS & $\mathrm{R}$ & $\mathrm{R}^{2}$ & $\mathrm{~F}$ & Sig.(F) & BETA & $t$ & $p$ \\
\hline Psicológico & 0,515 & 0,265 & 11,16 & 0,002 & 0,394 & 2,94 & 0,006 \\
CD4 & 0,639 & 0,408 & 10,34 & 0,0001 & 0,420 & 3,18 & 0,003 \\
Ambiental & 0,715 & 0,511 & 10,09 & 0,0001 & 0,328 & 2,46 & 0,02
\end{tabular}

* significativo a $p \leq 0,05$

\section{Discussão}

O perfil sócio-econômico dos participantes confirmou a tendência nacional de pauperização da epidemia (Brasil, 2010; Parker \& Camargo, 2000). De modo geral, o perfil sociodemográfico e clínico da amostra são semelhantes às observadas nos pacientes adultos com HIV/AIDS no Brasil e em outros países: amostra com maioria masculina, com baixa renda e baixa escolaridade, uso da HAART e baixa frequência de doenças concomitantes ao HIV. A convivência com uma patologia crônica demanda cuidados médicos e o uso continuado de medicamentos, contribuindo para a necessidade de suporte financeiro que, quando da sua ausência, pode ser um fator de estresse para as pessoas. Não obstante, os resultados apontaram uma percepção geral de qualidade de vida satisfatória, bem como avaliações positivas em todos os domínios medidos.

Neste estudo, verifica-se que a baixa renda apresenta-se como variável que pode dificultar a vivência cotidiana no lidar com a infecção, tendo em vista que os participantes mencionaram a dimensão ambiental - presença de maiores recursos financeiros, qualidade na assistência à saúde, melhores informações sobre a sua infecção, etc. - como relevante para uma avaliação de qualidade de vida satisfatória, mas que, por si, não determina a avaliação geral de qualidade de vida. Além disso, os participantes afirmaram estar "muito" ou "bastante satisfeitos" com os serviços de saúde que utilizavam, evidenciando-se que os usuários mais satisfeitos com os serviços de saúde, em comparação com os usuários pouco satisfeitos, apresentaram médias mais satisfatórias em sua qualidade de vida geral e nos domínios físico, psicológico e ambiental. Embora esses achados sejam descritivos, são importantes, pois uma maior qualidade nos serviços de saúde tem sido evidenciada como um fator relevante na adesão ao tratamento do HIV/AIDS (Gupta, Silva, \& Passos, 2005).

No que se refere aos escores de qualidade de vida, eles são uma escala positiva (quanto maior o escore, melhor a qualidade de vida), e não existem pontos de corte que determinem um escore abaixo ou acima do qual se possa avaliar a qualidade de vida como "ruim" ou "boa", no entanto, neste estudo tomou-se o escore 50 como ponto de corte. Neste sentido, os resultados deste estudo indicam que os participantes apresentaram bons escores de qualidade de vida para todos os domínios do WHOQOLBREF, quando comparados à pontuação máxima dos escores para cada domínio e às médias encontradas para os diferentes domínios da qualidade de vida na população estudada. Dentre os domínios, destaca-se o psicológico como melhor avaliado, indicando que estes participantes apresentam enfrentamento positivo frente às adversidades impostas, provavelmente derivadas ou derivando da/na avaliação positiva satisfatória referente ao atendimento no serviço de saúde. O domínio com menor média foi o ambiental, concernente com as condições estruturais de vida apresentada, que não necessariamente são causadas pela situação de adoecimento, parecendo ser anterior a ela.

Quando se estudou a influência das variáveis sociodemográficas nos diferentes domínios da qualidade de vida, observou-se que somente a variável sexo teve influência significativa no domínio físico, sendo os escores médios desses domínios significativamente maiores entre as mulheres. Este resultado pode estar associado aos aspectos de gênero, a partir dos quais condições estruturais semelhantes podem ter formas diferentes de análise e ponderação. Neste sentido, a avaliação do domínio físico (dor, desconforto, energia, fadiga, sono, repouso, mobilidade, atividades da vida cotidiana, dependência 
de medicação ou de tratamentos e capacidade para o trabalho) poderia estar relacionada à capacidade funcional como importante fator de impacto na qualidade de vida, associado à atividade laboral, tão importante na constituição da identidade masculina ou mesmo sua falta, - o desemprego/a não ocupação - (Schraiber, Gomes, \& Couto, 2005), o que poderia explicar a avaliação mais negativa.

$\mathrm{Na}$ análise da associação dos dados clínicos com a qualidade de vida, verificou-se que as pessoas com contagem de linfócitos CD4 acima de $500 \mathrm{~mm}^{3}$ avaliaram melhor a sua qualidade de vida geral em comparação com os participantes com uma contagem abaixo de $500 \mathrm{~mm}^{3}$, sugerindo que a boa avaliação da progressão clínica do HIV esteve associada com melhores indícios psicossociais. A relevância da estimativa da contagem de células CD4 em pacientes com HIV/AIDS se dá pelo reconhecimento dessas células como importantes preditores biológicos do status da doença e sua progressão, bem como de vulnerabilidade a infecções oportunistas (Remor, et al., 2007; Zimpel \& Fleck, 2008).

O estudo de Santos, Franca Junior e Lopes (2007) corrobora também esses resultados. Em um serviço ambulatorial de atendimento a pessoas vivendo com HIV/AIDS, utilizando-se o WHOQOL-BREF, verificou-se que, dentre os principais fatores sócio-demográficos e clínicos relacionados com qualidade de vida, destacaram-se: a contagem de células CD4, presença de sintomas clínicos e tempo conhecido de infecção pelo HIV. Dessa forma, a contagem de células CD4 torna-se relevante na avaliação de uma qualidade de vida satisfatória, tendo em vista que esse construto engloba também as dimensões físicas e de cuidado com a saúde, as quais influenciam na percepção de saúde-doença.

Ao analisar a contribuição dos diferentes domínios e variáveis na qualidade de vida global, observou-se a explicação conjunta de $51 \%$ diferindo a respeito da contribuição individual na qualidade de vida global: o domínio que mais contribuiu na qualidade de vida global foi o psicológico, seguido do CD4 e do ambiental. Achados nesta direção têm sido apontados em estudos que versam sobre a relação entre os aspectos psicossociais, clínicos e avaliação de qualidade de vida. Em estudo realizado com homens e mulheres que viviam com HIV/AIDS, controlando-se as variáveis sociodemográficas e clínicas, verificou que o estresse percebido se relacionou com a diminuição de células CD4 (Remor, et al., 2007; Zeña, et al., 2009). As dimensões psicossociais também foram evidenciadas em outro estudo, onde se verificou que altos níveis de ansiedade e depressão associados à hospitalização em pacientes com AIDS se relacionaram com uma diminuição do número de células de CD4, ressaltando-se a importância de aspectos psicoafetivos para a melhor vivência da soropositividade ao HIV/AIDS (Zeña, et al., 2009).

Faz-se importante ressaltar a grande variabilidade no tempo de diagnóstico da infecção, revelando o aumento na expectativa de vida dos participantes, aspecto esse que pode estar associado ao uso da terapia antirretroviral mencionada pela maioria das pessoas $(88 \%)$. Nesse sentido, o aumento da expectativa de vida, relacionada pela presença dos medicamentos antiretrovirais leva a mudanças na maneira de enfrentamento da infecção pelo
HIV, pois vem proporcionar a extinção de uma representação da doença equivalente à morte, característico do início da epidemia (Almeida \& Labrinici, 2007). Em virtude disso, muitas pessoas vivendo com AIDS têm recuperado sua capacidade de se relacionar, interagir, amar e trabalhar em sociedade, o que tem proporcionado uma melhor avaliação de suas vidas (Ayres, 2002). Esses aspectos têm levado a mudanças no foco de estudos em HIV/AIDS, o qual estava inicialmente concentrado na incidência de doenças oportunistas e transtornos mentais. Tais mudanças têm levado em conta fatores que promovem bem-estar psicológico, presença e redução de preconceito em relação à infecção, aderência ao tratamento e, principalmente, avaliação de qualidade de vida no contexto do HIV/AIDS (Almeida \& Labrinici, 2007; Meng et al., 2008; Seidl, 2005).

Por fim, observou-se que a variável qualidade de vida geral está correlacionada aos domínios, que, por sua vez, se encontram correlacionados entre si. Isto leva à constatação de que a qualidade de vida, assim como as demandas em saúde, não se restringe às demandas biológicas, tampouco podem ser consideradas como individuais e isoladas, ao contrário, sob a perspectiva da Saúde Coletiva, as necessidades devem estar articuladas às necessidades sociais, que são heterogêneas e originam-se da reprodução da vida em sociedade (Alves, 2005). Cada trajetória pessoal e psicossocial depende dos contextos intersubjetivos e das relações de poder que podem ser entendidas apenas em termos de seus significados locais e contextos estruturais. Desta forma, conforme Pereira et al (2008), a interpretação da avaliação global da qualidade de vida é um tanto difícil, uma vez que resultados semelhantes podem ser explicados por diferentes fatores. Para cada indivíduo, há uma forma de operacionalizar sua avaliação, e a avaliação de um mesmo indivíduo pode variar com o tempo, com a variação de prioridades ao longo da vida e com as circunstâncias pelas quais a vida pode se modificar.

\section{Considerações finais}

A partir deste estudo, ressalta-se a preponderância dos aspectos psicossociais na vivência de pessoas soropositivas ao HIV/AIDS. Destaca-se que independentemente das condições econômicas e sociais, a percepção de qualidade de vida tem sido caracterizada como positiva, levando-se em consideração outros determinantes para uma percepção de vida satisfatória, como, por exemplo, os elementos psicossociais e clínicos.

Nesse sentido, a constatação da presença das dimensões psicológica, imunológica e meio ambiente para uma percepção de qualidade de vida satisfatória revela a importância das redes de apoio psicossocial no cuidado de pessoas que vivem com HIV/ AIDS. Além disso, os fatores supracitados trazem a necessidade um cuidado mais integral às pessoas que vivem com HIV/AIDS. Igualmente, ressalta-se a importância da avaliação imunológica no prognóstico clínico e vivência subjetiva da infecção, pois essa avaliação pode estar relacionada também com uma avaliação satisfatória de saúde.

Como limitações do estudo, cita-se o delineamento transversal que impede a determinação de uma relação causa/ efeito entre as associações. Reconhece-se, também, a necessidade 
de avaliar de forma mais precisa o nível de adesão ao tratamento antirretroviral, tendo em vista que essa variável pode ser importante na avaliação do tratamento e progressão clínica da infecção. Do mesmo modo, se identifica a necessidade de uma análise mais aprofundada entre tempo de diagnóstico e percepção de qualidade de vida. Achados dessa ordem poderiam trazer maior entendimento sobre de que forma variáveis psicossociais se manifestam no maior prolongamento de vida de pessoas com HIV/AIDS. Por outro lado, uma metodologia de pesquisa qualitativa em qualidade de vida abordaria as vivências subjetivas da AIDS, assim como uma maior descrição dos determinantes psicossociais no cotidiano de pessoas vivendo com HIV/AIDS.

Finalmente, evidencia-se a necessidade de mais estudos que possam avaliar de forma mais clara a relação entre aspectos clínicos, psicológicos e ambientais na percepção de qualidade de vida entre pacientes que vivem com HIV/AIDS. Dessa forma, sugere-se a investigação sobre a maneira como os determinantes psicossociais estão influenciando e sendo influenciados pelo determinante imunológico no contexto do HIV/AIDS.

\section{Referências}

Almeida, M. R. C. B., \& Labrinici, L. M. (2007). A trajetória silenciosa de pessoas portadoras de HIV contada pela história oral. Ciência \& Saúde Coletiva, 12(1), 263-274.

Alves, V. S. (2005). Um modelo de educação em saúde para o Programa Saúde da Família: pela integralidade da atenção e reorientação do modelo assistencial. Interface: Comunicação, Saúde, Educação, 9(16), 39-52.

Ayres, L. R. C. M. (2002). Educational practices and the preventions of HIV/ AIDS: Lessons learned and current challenges. Interface: Comunicação, Saúde e Educação, 6(11), 11-24.

Brasil, Ministério da Saúde (1996). Resolução 196/96 sobre pesquisa envolvendo seres humanos. Brasília: Conselho Nacional de Saúde - CNS.

Brasil, Ministério da Saúde (2005). Adesão ao Antirretroviral-manual para profissionais de saúde. Brasília: Ministério da Saúde, Programa Nacional de HIV/AIDS. Recuperado de http://www.saude.rio.rj.gov.br/media/ dstAIDS_adesao_ms_2005.pdf

Brasil, Ministério da Saúde. (2010). Resposta Brasileira 2008 - 2009. Relatório de Progresso do País. Recuperado de http://www.AIDS.gov.br

Chatterji, S., \& Bickenbach, J. (2008). Considerações sobre a qualidade de vida. In M. P. A. Fleck (Org.), A avaliação de qualidade de vida: Guia para profissionais da saúde. (pp. 40-47) Porto Alegre: Artmed.

Fleck, M. P. A. (2008). Problemas conceituais em qualidade de vida. In M. P. Fleck. (Org.), A avaliação de qualidade de vida: Guia para profissionais da saúde. (pp. 19-28). Porto Alegre: Artmed.

Fleck, M. P. A., Leal, O. F., Louzada, S., Xavier, M., Chachamovich, E., Vieira, ... Pinzon, V. (1999). Desenvolvimento da versão em português do instrumento de avaliação de qualidade de vida da OMS (WHOQOL-100). Revista Brasileira de Psiquiatria, 21(1)19-28.

Guimarães, C. D. (2001). AIDS no feminino: Por que a cada dia mais mulheres contraem AIDS no Brasil? Rio de Janeiro: Editora UFRJ.

Gupta, N., Silva, A. C. S., \& Passos, L. N. (2005). The role of integrated homebased care in patient adherence to antiretroviral therapy. Revista da Sociedade Brasileira de Medicina Tropical, 38(3), 241-245.

Ironson, G., O'Cleirigh, C., Fletcher, M. A., Laurenceau, J. P., Balbin, E., Klimas, ... Solomon, G. (2005). Psychosocial factors predict CD4 and viral load change in men and women with human immunodeficiency virus in the era of highly active antiretroviral treatment. Psychosomatic Medicine, 67(1), 1013-1021.doi:10.1097\%2F01.psy.0000188569.58998.c8

McCutchan, J. A. A., Wub, J. W., Robertson, K., Koletard, S. L., Ellisa, R. J., Cohn, S., ... Williams, P. L. (2007). HIV suppression by HAART preserves cognitive function in advanced, immune-reconstituted AIDS patients. AIDS: Official Journal of the International AIDS Society, 21(9), 1109-1117. doi: 10.1097/QAD.0b013e3280ef6acd

Meng, Y. J., Li, N. X., Liu, C. J., Chen, J. H, Song, Y. C., \& Qian, Z.S. (2008). Quality of life and hostile mentality trend of patients with HIV/AIDS in China. The Royal Institute of Public Health, 122, 404-411.

Panzini, R. G., Rocha, N. S., Bandeira, D. R., \& Fleck, M. P. A. (2008) Espiritualidade/religiosidade e qualidade de vida. In M. P. A. Fleck (Org.), A avaliação de qualidade de vida. Guia para profissionais de saúde (pp. 177-96). Porto Alegre: Artmed.

Parker, R., \& Camargo Jr., K. R. (2000). Pobreza e HIV/AIDS: Aspectos antropológicos e sociológicos. Cadernos de Saúde Pública, 16(1), 89-102.

Pereira, R. J.; Cotta, R. M. M.; Franceschini, S. C. C., Ribeiro, R. C. L.; Sampaio, R. F.; Priore, S. E., \& Cecon, P. R. (2006). Contribuição dos domínios físico, social, psicológico e ambiental para a qualidade de vida global de idosos. Revista de Psiquiatria do Rio Grande do. Sul, 28(1), 27-38.

Ravagnani, L. M. B., Domingos, N. A. M., \& Miyazaki, M. C. O. S. (2007) Qualidade de vida e estratégias de enfrentamento em pacientes submetidos a transplante renal. Estudos de Psicologia (Natal), 12(2), 177-184.

Remor, U. S A. (2002). Psiconeuroimunología e infecção por HIV: Realidade ou ficção? Psicologia: Reflexão e Crítica, 15(1), 113-119.

Remor, E., Penedo, F. J., Shen, B-J., \& Schneiderman, N. (2007). Perceived stress is associated with CD4+ decline in men and women living with HIV/AIDS in Spain. AIDS Care: Psychological and Socio-medical Aspects of AIDS/ HIV, 19(2), 215-219.

Santos, E. C. M., Franca Junior, I., \& Lopes, F. (2007). Qualidade de vida de pessoas vivendo com HIV/AIDS em São Paulo. Revista de Saúde Pública, 41(2), 64-71.

Schraiber, L.B.; Gomes, R., \& Couto, M.T. (2005). Homens e saúde na pauta da saúde coletiva. Ciência \& Saúde Coletiva, 10(1): 7-17.

Seidl, E. M. F. (2005). Enfrentamento, aspectos clínicos e sociodemográficos de pessoas vivendo com HIV/AIDS. Psicologia em Estudo, 10(3), 421-429.

Seidl, E. M. F., Melchíades, A., Farias, V., \& Brito, A. (2007). Pessoas vivendo com HIV/AIDS: Variáveis associadas à adesão ao tratamento anti-retroviral. Cadernos de Saúde Pública, 23(10), 2305-2316.

Seidl, E. M. F., Zannon, C. M. L. C., \& Trócolli, B. T. (2005). Pessoas vivendo com HIV/AIDS: enfrentamento, suporte social e qualidade de vida. Psicologia Reflexão e Crítica, 18(2), 188-195.

The WHOQOL Group. (1995). The world health organization quality of life assessment (WHOQOL): Position paper from the World Health Organization. Social Science \& Medicine, 41(1), 1403-1410.

Torres, M. A., \& Oliveira, A. C. P. (2004). Distúrbios cognitivos e demência associados ao HIV. In Ministério da Saúde: Secretaria da Vigilância em Saúde. Programa Nacional de DST e AIDS (Org.), Manual de Assistência psiquiátrica em HIV/AIDS (pp. 57-61). Brasília: Ministério da Saúde.

Zeña C., D., Mezones H., E., Valdiviezo G., G., La Chira A., A., Rodriguez M., J., \& Dickson G., S. (2009). Impact of hospital-associated anxiety and depression on the CD4 counts of naïve HIV/AIDS patients from locations in Northern Peru. International Journal of Infectious Diseases, 13(2), 75-76.

Zimpel, R., \& Fleck, M. P. A. (2008). WHOQOL-HIV: Desenvolvimento, aplicação e validação. In M. P. A. Fleck (Org.), A avaliação de qualidade de vida: Guia para profissionais de saúde (pp. 83-92). Porto Alegre: Artmed. 
Bruno Medeiros, Mestre em Psicologia pela Universidade Federal da Paraíba (UFPB), Doutorando em Psicologia pela Universidade de Cambridge, Reino Unido. Endereço para correspondência: Rua Antônio Lustosa Cabral, 141, Cabo Branco. João Pessoa - Paraíba, CEP: 58045020. Telefone: (83) 356-68398 e (44) 07848 947199. Email: brunojpa@hotmail.com

Josevânia da Silva, Doutora em Psicologia Social pela Universidade Federal da Paraíba (UFPB), é professora titular no Centro Universitário de João Pessoa (UNIPE). Email: josevaniasco@gmail.com

Ana Alayde Werba Saldanha, Pós-doutora e Doutora em Psicologia pela Universidade de São Paulo (USP), é professora associada I na Universidade Federal da Paraíba (UFPB). Email:

analayde@gmail.com 